\title{
McArdle syndrome with recurrent rhabdomyolysis in a middle aged man
}

\author{
H M A Ediriweera ${ }^{1}$, G G A Gayani ${ }^{1}$, P D W Dilhani ${ }^{1}$, K D Pathirana ${ }^{2}$, T P Weerarathna ${ }^{2}$ \\ Ceylon Medical Journal 2014; 59: 99-100
}

\section{Introduction}

McArdle syndrome (glycogen storage disease type $\mathrm{V}$ ) is a metabolic myopathy causing exercise induced rhabdomyolysis due to deficiency of muscle phosphorylase. It is an autosomal recessive disorder, presenting during childhood with symptoms related to musculoskeletal system. We report a case of McArdle syndrome presenting with recurrent rhabdomyolysis.

\section{Case report}

A 44-year old male estate labourer presented with severe muscle pains and passing dark urine of two days duration. Six hours prior to the onset of symptoms, he sustained a fall from height of about six feet. The muscle pains progressively worsened over the next two days and he was almost immobilised due to severe generalised muscle pain by the second day of the illness. On the same day, he noticed that his urine was dark brown in colour and he was passing smaller quantities of urine than before. He gave a past history of similar episode following a febrile illness with acute kidney injury two years ago. He recovered after six cycles of haemodialysis. He had two other similar episodes during the last two years but these were milder than the first episode. One of them was precipitated by strenuous physical activity and the other was subsequent to an alcohol binge.

On examination, he was an average-built man with a BMI of $21 \mathrm{~kg} / \mathrm{m}^{2}$. Musculoskeletal system examination showed mild degree of muscle wasting and weakness of both upper and lower limbs bilaterally, with the proximal muscles being affected more than the distal muscles. Deep tendon reflexes were diminished. The weakness and reflexes improved towards the latter part of the illness. Rest of the system examination was normal.

Investigation findings confirmed rhabdomyolysis. Serum creatine phosphokinase (CPK) 4170 units/l (39-308) and urine myoglobin was positive. Ischaemic forearm exercise test (IFET) showed poor lactate response with less than two fold rise in lactate as shown in Figure 1.
Electromyogram (EMG) showed resting fibrillating potentials with smaller amplitude motor units. Muscle biopsy from right quadriceps showed degenerating and regenerating muscle fibers and minimal inflammation with haematoxylin and eosin (H \& E) stain (Figure 2), while Periodic Acid Schiff (PAS) stain showed subsarcolemmal intense staining of muscle glycogen (Figure 3). The facilities for histochemical analysis of muscle biopsy for phosphorylase activity were not available for further confirmation.

Lactate $\mathrm{mmol} / 1$

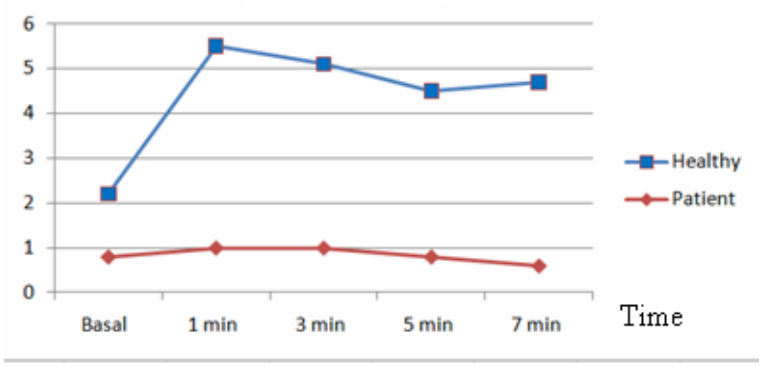

Figure 1. Ischaemic forearm exercise test.

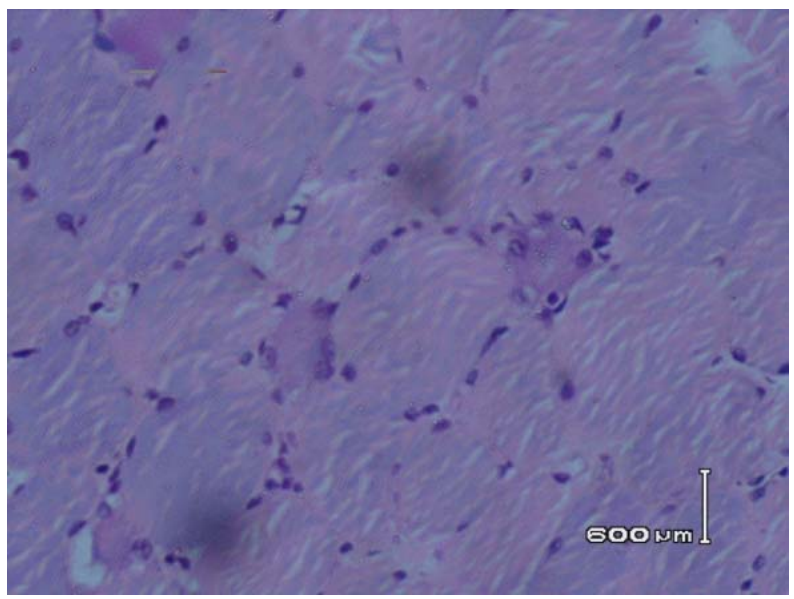

Figure 2. Biopsy H and E Stain.

${ }^{1}$ University Medical Unit, Teaching Hospital Karapitiya, Galle and ${ }^{2}$ Department of Medicine, Faculty of Medicine, University of Ruhuna, Galle, Sri Lanka.

Correspondence: HMAE, e-mail: <anushkaediriweera@gmail.com>. Received 20 February 2014 and revised version accepted 25 May 2014. Competing interests: none declared. 


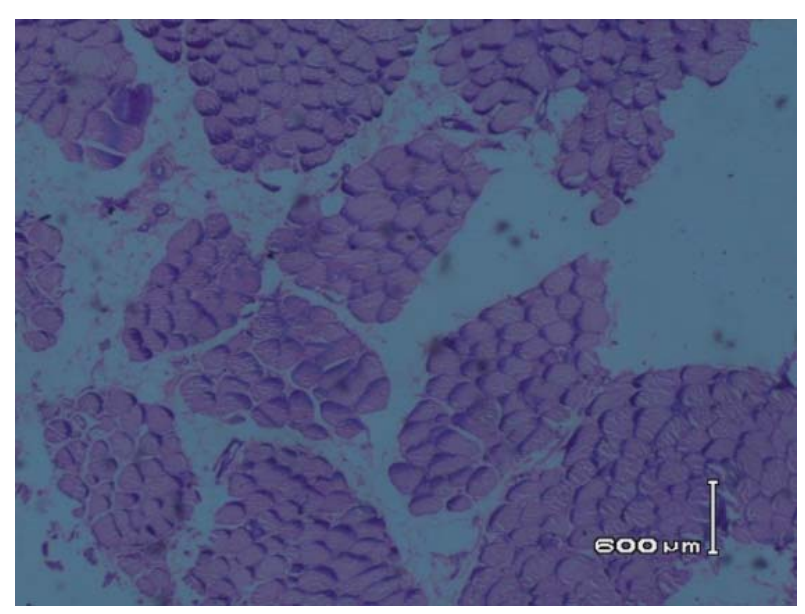

Figure 3. Muscle biopsy PAS Stain.

The diagnosis of McArdle syndrome was made based on clinical, ischaemic forearm exercise test, EMG findings and muscle histology. He was managed with supportive care. Advice was given about preventing further episodes of rhabdomyolysis. His disability was improved with time though the CPK levels were persistently elevated even two months after the acute event (3115 U/l).

\section{Discussion}

McArdle syndrome is caused by muscle phosphorylase deficiency [1]. The enzyme is responsible for breaking down of glycogen in muscle and its deficiency results in defective glycogenolysis and glycogen accumulation in muscle. Consequently, poor energy production in skeletal muscle during muscular activity will result in muscle damage. Extensive muscle damage produces symptoms of rhabdomyolysis. Affected individuals present in adolescence or early adulthood with exercise intolerance, myalgia, cramps, myoglobinuria, muscle swelling, and fixed weakness. Some patients present with episodes of myoglobinuria with acute kidney injury [2]. Late presentation of symptoms has been reported [3].

The IFET is usually diagnostic of phosphorylase deficiency, showing a flat venous lactate curve. Non ischaemic forearm test is safer than ischaemic test and it is as accurate [4]. The muscle biopsy in phosphorylase deficiency may show focal subsarcolemmal and intermyofibrillar accumulations ("blebs") of normally structured glycogen. Negative histochemical reaction for phosphorylase activity of muscle frozen section confirms the diagnosis.

Muscle biopsy taken just after an acute event of rhabdomyolysis may give false positive results because of the existence of fetal isozyme in the regenerating muscle fibers. The resting EMG in patients with muscle phosphorylase deficiency is abnormal; it shows resting fibrillating potentials and myopathic changes (small amplitude motor units) on contraction [3].

There is no specific treatment for this condition. Potential therapies include relevant dietary modification, moderate aerobic exercise, and supplementation with creatine and vitamin B6. Ingestion of sucrose five minutes before all types of dynamic exercise is advised [5]. Moderate physical activity and low-level warm-up before any planned moderate exercise would reduce the risk of exercise induced rhabdomyolysis. General anesthesia and statin therapy should be used with caution in these patients as those might precipitate an acute event with myoglobinuria.

Recurrent rhabdomyolysis even in middle age, without an obvious cause needs to be evaluated to exclude rare disorders like McArdle syndrome because precautions can minimise recurrences and life threatening complications.

\section{References}

1. Dan Longo, Anthony Fauci, Dennis Kasper et al. Glycogen Storage Diseases and Other Inherited Disorders of Carbohydrate Metabolism. In: Harrison's Internal Medicine, 18th ed. Chapter 362. McGraw Hill, 2012; 3202.

2. Joaquin Arenas, Miguel A Martin, Antonio L Andreu. In: Pagon RA, Adam MP, Bird TD, et al. Glycogen Storage Disease Type V. Gene Reviews, 2006.

3. Pourmand R, Sanders DB, Corwin HM. Late onset McArdle's disease with unusual electromyographic findings. Archives of Neurology 1983; 40: 374-7.

4. Kazemi-Esfarjani P, Skomorowska E, Jensen TD, Haller RG, Vissing J. A nonischaemic forearm exercise test for McArdle disease. Annals of Neurology 2002; 52: 153-9.

5. Vissing J, Haller RG. The effect of oral sucrose on exercise tolerance in patients with McArdle's disease. New England Journal of Medicine 2003; 349: 2503-9. 